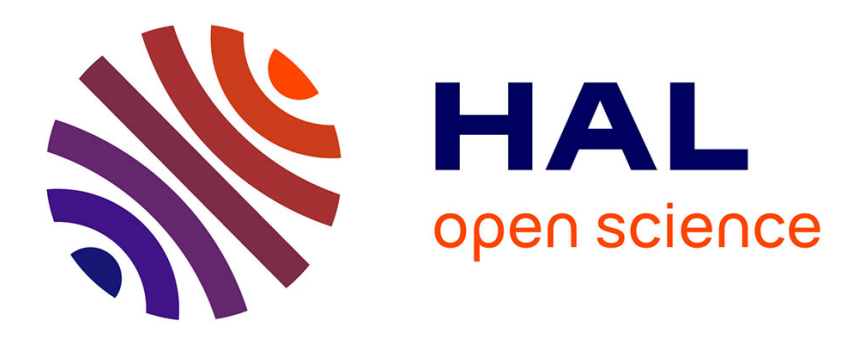

\title{
La manipulation du résultat comptable avant une offre publique
}

Arnaud Thauvron

\section{To cite this version:}

Arnaud Thauvron. La manipulation du résultat comptable avant une offre publique. Comptabilité Contrôle - Audit, 2000, 6, pp.97 - 114. 10.3917/cca.062.0097 . hal-01386324

\section{HAL Id: hal-01386324 \\ https://hal.science/hal-01386324}

Submitted on 23 Oct 2016

HAL is a multi-disciplinary open access archive for the deposit and dissemination of scientific research documents, whether they are published or not. The documents may come from teaching and research institutions in France or abroad, or from public or private research centers.
L'archive ouverte pluridisciplinaire $\mathbf{H A L}$, est destinée au dépôt et à la diffusion de documents scientifiques de niveau recherche, publiés ou non, émanant des établissements d'enseignement et de recherche français ou étrangers, des laboratoires publics ou privés. 


\section{LA MANIPULATION DU RÉSULTAT COMPTABLE AVANT UNE OFFRE PUBLIQUE}

Arnaud Thauvron

Association francophone de comptabilité | «Comptabilité - Contrôle - Audit »

2000/2 Tome 6 | pages 97 à 114

ISSN 1262-2788

ISBN 2711734129

Article disponible en ligne à l'adresse :

http://www.cairn.info/revue-comptabilite-controle-audit-2000-2-page-97.htm

\section{Pour citer cet article :}

Arnaud Thauvron, « La manipulation du résultat comptable avant une offre publique », Comptabilité - Contrôle - Audit 2000/2 (Tome 6), p. 97-114.

DOI 10.3917/cca.062.0097

Distribution électronique Cairn.info pour Association francophone de comptabilité.

(C) Association francophone de comptabilité. Tous droits réservés pour tous pays.

La reproduction ou représentation de cet article, notamment par photocopie, n'est autorisée que dans les limites des conditions générales d'utilisation du site ou, le cas échéant, des conditions générales de la licence souscrite par votre établissement. Toute autre reproduction ou représentation, en tout ou partie, sous quelque forme et de quelque manière que ce soit, est interdite sauf accord préalable et écrit de l'éditeur, en dehors des cas prévus par la législation en vigueur en France. Il est précisé que son stockage dans une base de données est également interdit. 


\section{La manipulation du résultat comptable avant} une offre publique

\section{Arnaud Thauvron}

\section{Résumé}

Cette étude teste I'hypothese selon laquelle les dirrigeants des sociétés quil lancent une offre publique sur l'une de leurs filiales réduisent le résultat comptable de cette dernière. L'objectif poursuivi est de minimiser le prix d'offre. Un examen de 95 offres publiquues met en évídence une baisse des résultats avant loffre: Aucune manipulation des variables comptables de régularisation n'est cependant observée. Trois hypotheses alternatives sont alors proposées.

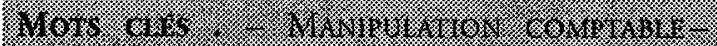

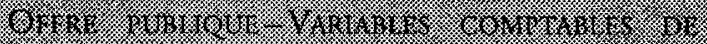

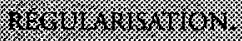

\section{Abstrod I Earnings management before a takeover \\ This study tessts the hypothessis that manugers of firms that are bidders of takeovers on a subsi- diary tedice reported earnings of the target. The objective pursued is to minimize the offer price. An examination of 95 tender offers provides evi. dence of eamings decrease. No manipulation of accruals is observed. Three alternative bypothesis are proposed.}

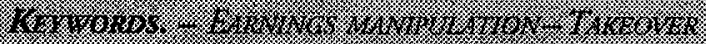

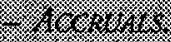

Correspondance :

\begin{abstract}
Arnaud THAuvron
École supérieure des affaires -IRG

Université Paris XII-Val-de-Marne

e.mail : thauvron@univ-paris12.fr
\end{abstract}

\section{Introduction}

La manipulation du résultat comptable d'une société a pour objet de faire parvenir au marché un signal erroné. Ce signal conduit les agents à réviser leurs anticipations quant aux flux de trésorerie futurs de la société, ce qui en modifie la valorisation par le marché, valorisation qui est alors différente de la valeur intrinsèque. Cette manipulation présente notamment un intérêt lors des offres publiques de renforcement du contrôle et de fermeture du capital, opérations dans lesquelles l'initiateur de l'offre est l'actionnaire majoritaire de la cible. Ces offres, offres publiques simplifiées (OPS), de retrait 
(OPR) ou suivies d'un retrait obligatoire (RO) représentent, au cours de la période 1993-1997, $64 \%$ du nombre total des opérations.

Dans ce type d'opération, l'initiateur de l'offre dispose d'un avantage informationnel quant à la valeur intrinsèque de la société cible du fait d'un accès complet à l'information. Ceci engendre une asymétrie de l'information entre l'initiateur et les actionnaires minoritaires. Ce problème est accentué par le conflit d'intérêt que peut engendrer l'opération. En tant que dirigeant, l'initiateur a un devoir fiduciaire à l'égard des autres actionnaires afin de négocier un prix d'offre qui soit le plus élevé possible. Mais, dans le même temps, sa situation personnelle d'initiateur d'une offre publique l'incite à offrir un prix qui soit relativement bas afin de maximiser sa richesse personnelle (DeAngelo et al., 1984). Ce phénomène s'est fortement accentué avec l'introduction de la procédure de retrait obligatoire en décembre 1993, à l'issue de laquelle les minoritaires sont expropriés de leurs titres au profit de l'actionnaire majoritaire. Cette position d'actionnaire majoritaire peut permettre de gérer le résultat comptable de la filiale avec un double objectif : influer sur le résultat de la mise en ouvre des méthodes d'évaluation fondées sur des données comptables et faire parvenir au marché un signal erroné qui induise une baisse des cours. Les différentes évaluations de la cible ainsi minorées, il lui est possible de proposer un prix d'offre réduit. Cette hypothèse est d'autant plus plausible que si les termes de l'opération ne sont pas satisfaisants, aucune régulation par le marché n'est envisageable car une contre-offre est impossible.

Ce conflit d'intérêt a conduit à la mise en place d'expertises indépendantes, sans pour autant atténuer les plaintes des actionnaires minoritaires. En effet, la mission de l'expert indépendant n'est en aucun cas de contrôler les informations comptables qui servent à l'évaluation, cette mission étant dévolue au commissaire aux comptes. Sa mission est de porter une appréciation sur la pertinence des méthodes d'évaluation ainsi que sur les options retenues. $\AA$ son tour, il fondera son analyse du prix proposé sur les données comptables de la cible.

L'objet de cette recherche est de tester l'hypothèse selon laquelle les initiateurs d'une offre publique, lorsqu'ils détiennent préalablement le contrôle de la cible, sont incités à manipuler le résultat comptable de leur filiale afin de proposer un prix d'offre réduit.

Dans une première section, la théorie positive de la comptabilité est présentée ainsi que l'intérêt que peut offrir une manipulation du résultat dans le cadre d'une offre publique. La deuxième section expose les résultats d'études antérieures, qu'elles soient relatives aux offres publiques ou au Management Buy Out. La troisième section est consacrée à une validation empirique de l'hypothèse avancée, sur un échantillon de 95 offres publiques. Les résultats sont présentés dans une quatrième et dernière section.

\section{La théorie positive de la comptabilité et les marchés boursiers}

Selon la théorie positive de la comptabilité, les dirigeants de sociétés optent pour des procédures comptables soit de façon efficiente, dans le but de maximiser la richesse des actionnaires, soit de façon opportuniste pour maximiser leur propre richesse ' . Pour Cormier et Magnan (1995), " la gestion stratégique des résultats peut se définir comme une intervention de la part de la direction d'une firme 
dans le processus de présentation de l'information financière en vue d'obtenir un gain au détriment d'autres parties ». Le sens de la gestion dépend alors des contrats implicites ou explicites qui lient la société et ses dirigeants. Cette théorie explique les choix en matière de politique comptable à partir de l'hypothèse selon laquelle ils sont guidés par un objectif de maximisation de l'utilité, que ce soit celle des actionnaires ou celle des dirigeants (Raffournier, 1990). Ces choix comptables résultent des relations d'agence qui existent au niveau de chaque entreprise. La part relative du comportement efficient des dirigeants par rapport aux procédures opportunistes sera fonction du niveau de contrôle qui est exercé sur ces derniers par les différents mécanismes disciplinaires (Christie et Zimmerman, 1994). Ces mécanismes sont notamment le conseil d'administration, le marché du contrôle ou celui des équipes dirigeantes. En intégrant la théorie de l'agence et la théorie des coûts politiques, la recherche positive a ainsi mis en évidence le fait que les choix comptables étaient plus motivés par des comportements opportunistes que par le seul désir de " donner une image fidèle " ${ }^{2}$ (Dumontier et Raffournier, 1999). Pour autant, plusieurs études ont montré que la gestion du résultat permettait à ce dernier de mieux refléter la valeur des sociétés (Subramanyam, 1996). La corrélation entre la performance boursière et le résultat comptable est en effet plus élevée qu'avec les flux de trésorerie.

L'étude de la gestion des résultats suppose qu'au préalable soit défini le caractère opportuniste ou efficient de cette gestion. Selon Christie et Zimmerman (1994), il y a opportunisme lorsque les décisions des dirigeants accroissent leur richesse propre sans que cela induise une création nette de richesse pour l'ensemble des participants (stakeholders). Une gestion des résultats est donc efficiente s'il y a création nette de richesse, et opportuniste si elle ne donne lieu qu'à un transfert de richesse au profit des dirigeants et au détriment des autres parties (actionnaires, créanciers, État, etc.). Dans le cas des offres publiques de renforcement et de fermeture, l'appréciation de la nature de la gestion comptable est rendue délicate par le fait qu'un comportement " opportuniste " pour les actionnaires de la cible se révèle être un comportement " efficient " pour ceux de l'initiateur. Dans le cadre de cette recherche, une gestion est considérée comme opportuniste dès lors qu'il y a transfert de richesse des actionnaires minoritaires de la cible au profit de ceux de l'initiateur, quand bien même il y aurait création nette de richesse du fait du rapprochement des deux sociétés ou de la disparition de certains coûts, tels que ceux de cotation.

La manipulation comptable des résultats de la cible a des conséquences tant sur le résultat de la mise en œuvre de certaines méthodes d'évaluation (méthodes analogiques) que sur son cours boursier.

\subsection{Les conséquences d'une manipulation comptable sur la valorisation de la cible}

Afin de minimiser le prix d'offre, l'initiateur-contrôlant peut avoir recours à une gestion appropriée du résultat comptable, en utilisant les options qui s'offrent à lui (durées et modes d'amortissement des immobilisations, méthodes d'évaluation des stocks, politique de provision, comptabilisation des charges financières, des frais de recherche et développement, etc.), à l'intérieur des principes comptables admis.

Une des conséquences de cette gestion est d'influer sur le résultat de la mise en ouvre des méthodes d'évaluation fondées sur les données comptables. En effet, lorsque le flottant du titre est faible, ce qui est le cas lors des OPR et des RO ainsi que lors de certaines offres publiques d'achat simplifiées (OPAS), il n'est plus possible de se référer uniquement au cours boursier qui a perdu de sa 
pertinence pour évaluer la cible. Il est alors nécessaire de mettre en œuvre des méthodes comparatives afin de valoriser l'entreprise. Ces méthodes consistent à appliquer à certains soldes de gestion de la société à évaluer un multiple obtenu à partir d'un panier d'entreprises comparables, notamment en termes d'activité. Ces soldes de gestion sont en particulier le résultat net (PER) et l'EBIT (résultat avant intérêts et impôts). Dès lors, en présence de manipulations, toute méthode fondée sur ce type de ratios comptables conduit à une sous-évaluation de la cible, lésant par là même les actionnaires minoritaires. Or, les méthodes d'évaluation qui font appel à des comparaisons sectorielles sont fréquemment utilisées lors des offres publiques (DeAngelo, 1986 et Thauvron, 1998).

\subsection{Les conséquences d'une manipulation comptable sur le cours boursier}

L'hypothèse d'efficience des marchés boursiers, dans sa forme semi-forte, implique que les cours s'ajustent immédiatement et sans biais à toute nouvelle information pertinente et représentent une bonne estimation de la valeur des titres. Cette hypothèse a notamment été étudiée dans le cadre de l'information comptable, et la relation entre les annonces de bénéfices et les cours boursiers a fait l'objet de très nombreuses études depuis les travaux de Ball et Brown (1968) et Beaver (1968). Patell et Wolfson (1984) montrent que les cours s'ajustent très rapidement aux annonces de bénéfices (entre 5 et 10 minutes), ce qui va dans le sens de l'hypothèse d'efficience des marchés boursiers. Toute nouvelle information comptable, si elle induit une révision des anticipations des agents, induit ainsi une modification des prix d'équilibre. La question qui se pose alors est de savoir si les investisseurs sont en mesure de détecter une éventuelle manipulation.

Teoh et al. (1998b) étudient la gestion des résultats avant une augmentation de capital. Ils mettent en évidence une relation entre une gestion comptable ex-ante et une mauvaise performance boursière ex-post. En gérant à la hausse le résultat, les dirigeants améliorent artificiellement les performances de la société et peuvent surévaluer le prix d'émission des nouveaux titres. Après l'opération, le niveau de performance ne peut être maintenu, ce qui conduit les investisseurs à réajuster leurs prévisions en fonction des fondamentaux de l'entreprise. Le marché n'est ainsi pas en mesure de détecter la manipulation comptable lors des émissions de nouveaux titres. Ces résultats sont confirmés par Ragan (1998) lors des augmentations de capital et par Teoh et al. (1998a) lors des introductions en Bourse. Pour autant, la manipulation du résultat peut aussi avoir pour objectif, non pas de faire parvenir au marché un signal erroné, mais de signaler aux investisseurs les anticipations des dirigeants qui ne sont pas nécessairement reflétées par les flux de trésorerie (Subramanyam, 1996 ; Degeorge, Patel et Zeckhauser, 1999). Subramanyam (1996) conclut cependant que sur un marché avec des asymétries informationnelles, une gestion du résultat peut être destinée à influer sur le cours boursier.

La publication de donnés comptables aura, par ailleurs, d'autant plus d'impact sur les cours boursiers que le marché dispose de peu d'informations. Or la majorité des sociétés qui sont la cible d'une offre de renforcement ou de fermeture ont un flottant relativement faible et font l'objet d'un suivi plus superficiel de la part des analystes financiers. En effet, la découverte d'un écart entre le cours et la valeur fondamentale d'un titre ne présente qu'un intérêt limité lorsque ce dernier est peu liquide car le gain monétaire qui peut en être retiré est faible, quand bien même il est important en pourcentage (Hamon, 1997). Dès lors, ces sociétés sont moins incitées à communiquer ${ }^{3}$ et la publication de leurs comptes annuels est souvent la principale (la seule) source d'information à destination du marché. 
L'importance de ce suivi par les analystes financiers a été mise en évidence par Chen, Lin et Sauer (1997) qui concluent que la qualité de l'information est avant tout fonction de la quantité d'information disponible, cette dernière étant proportionnelle au nombre d'analystes financiers suivant un même titre.

Dans le cas des cibles ayant un faible flottant, les investisseurs ne disposent que de peu d'éléments pour apprécier à sa juste valeur l'information comptable, d'où l'intérêt d'une gestion à la baisse des résultats. Ceci est renforcé par le fait que dans un nombre important de situations, les titres sont détenus directement par des particuliers et non par des professionnels (institutionnels, gestionnaires de portefeuilles, etc.), qui ne sont pas toujours à même de retraiter l'information (hypothèse de l'investisseur naif). Ceci s'explique par le fait que dans le cadre de leur activité, les gestionnaires de portefeuilles sont tenus à des impératifs de liquidité qui les conduisent à se concentrer sur les titres ayant un turn-over suffisant pour permettre de se retirer du marché au moindre coût.

La gestion des résultats a ainsi d'autant plus d'intérêt que l'information disponible est limitée et que le marché ne valorise pas correctement la société. L'écart entre le cours de Bourse et le prix proposé dans le cadre de l'offre apparaît alors d'autant plus élevé, donnant l'illusion qu'une prime importante est proposée aux minoritaires.

\section{La gestion des résultats lors des offres publiques et des MBO}

Plusieurs études se sont intéressées à la manipulation des résultats lors des offres publiques (Groff et Wright, 1989 ; Christie et Zimmerman, 1994 ; Easterwood, 1998 et Eddey et Taylor, 1999) ainsi que lors des $\mathrm{MBO}^{4}$ (DeAngelo, 1986 ; Perry et Williams, 1994 ; Wu, 1997 ; Le Nadant, 1999). Ces quatre dernières études sont plus proches de notre problématique que les premières, l'initiateur de la prise de contrôle étant un actionnaire de la société acquise.

\section{1. Études relatives aux offres publiques}

Pour Groff et Wright (1989), les sociétés qui font l'objet d'une prise de contrôle disciplinaire (offre publique) sont celles pour lesquelles la discipline par le marché du travail des équipes dirigeantes a échoué. Or, pour Fama (1980), ce type de discipline atténue les possibilités de gestion des résultats. Ceci conduit Groff et Wright à faire l'hypothèse que c'est dans les sociétés qui sont l'objet d'une prise de contrôle disciplinaire qu'il est probable d'observer une gestion des résultats (self-serving accounting choices).

Afin de détecter cette éventuelle gestion à la hausse des résultats, ils observent les changements de méthodes comprables des entreprises cibles d'une offre publique par rapport à un échantillon de contrôle. Ils font l'hypothèse que ces sociétés optent plus fréquemment que les autres pour l'évaluation de leurs stocks selon la méthode FIFO plutôt que LIFO, qu'elles préferent l'amortissement linéaire à l'amortissement dégressif et qu'elles utilisent une méthode particulière de comptabilisation du crédit d'impôt pour investissement. Leurs résultats sont en accord avec l'hypothèse selon laquelle les choix de politiques comptables sont affectés par l'environnement économique des dirigeants, 
ce qui les conduit à observer une utilisation plus fréquente des méthodes comptables qui augmentent le résultat.

Christie et Zimmerman (1994) reprennent les hypothèses de Groff et Wright (1989) et mesurent le comportement opportuniste des dirigeants en étudiant la fréquence d'utilisation des méthodes comptables augmentant le résultat par rapport à un échantillon de contrôle. Ils construisent des portefeuilles sectoriels afin de définir un niveau de choix efficient en matière comptable et d'estimer les choix opportunistes des sociétés cibles. Ils retiennent les trois même variables que Groff et Wright (1989) et aboutissent aux mêmes conclusions.

Avec une problématique quelque peu différente, Easterwood (1998) teste l'hypothèse selon laquelle les dirigeants de sociétés qui sont la cible d'une offre publique procèdent à une gestion à la hausse des résultats dans le but de faire échouer l'offre et de conserver leur emploi. La gestion des résultats peut, dans ce cas, être assimilée à une défense anti-OPA avec un objectif d'enracinement des dirigeants. Elle a pour finalité de convaincre les actionnaires que les dirigeants gèrent la société de façon efficace. La prime offerte serait alors trop faible et il serait de leur intérêt de ne pas apporter leurs titres à l'offre. Easterwood (1998) travaille sur un échantillon de 110 sociétés et utilise le modèle de DeAngelo (1986) ainsi qu'une version modifiée de celui de Jones (1991). En étudiant les résultats intermédiaires des cibles, elle met en évidence une gestion du résultat pendant le trimestre qui précède la tentative de prise de contrôle lorsque cette dernière est hostile et aucune manipulation dans le cas d'une offre amicale, ce qui sous-tend le fait que les dirigeants anticipent la survenance de l'offre. Concernant l'exercice qui suit l'annonce de l'offre, aucune gestion n'est décelée. Easterwood explique cette absence de manipulation par le fait que les dirigeants considèrent qu'il est désormais trop tard pour bénéficier des effets d'une gestion des résultats. Ces conclusions sont en accord avec DeAngelo (1988) qui observe une gestion à la hausse du résultat lors des batailles de mandats.

Sur le marché australien, Eddey et Taylor (1999) mettent en évidence des changements dans les variables comptables de régularisation (accruals) mais qui ne sont pas statistiquement significatifs.

\section{2. Études relatives aux $\mathrm{MBO}$}

DeAngelo (1986) s'intéresse aux choix comptables fait par les dirigeants de 64 sociétés cotées sur le NYSE ou l'AMEX et qui sont la cible d'un MBO entre 1973 et 1982. Bien que ces opérations fassent l'objet d'une expertise indépendante par une banque, elle considère que le conflit d'intérêt entre les dirigeants qui sont à l'initiative du MBO et les autres actionnaires n'est pas éteint, car les banquiers, tout comme la justice, mettent en œuvre des méthodes d'évaluation fondées sur les résultats comptables. Ceci peut inciter les dirigeants à minorer ces derniers afin de réduire le prix offert. À l'issue de son étude, elle ne parvient pas à mettre en évidence une gestion des résultats, rejetant l'hypothèse d'un comportement opportuniste des dirigeants.

Perry et Williams (1994) reprennent la même problématique que DeAngelo (1986) mais en utilisant une méthodologie plus robuste conceptuellement, fondée sur un modèle régressif, afin d'estimer la part du résultat qui est gérée. Leur étude retient 175 sociétés ayant fait l'objet d'un MBO entre 1981 et 1988 et montre une manipulation des résultats l'année précédant l'annonce de l'opération. Cette manipulation se fait aussi bien par le biais des dotations aux amortissements et provisions que par celui de la variation du besoin en fonds de roulement. En répliquant leur méthodologie sur l'échantillon de DeAngelo, ils n'observent aucune manipulation et concluent au fait que la différence 
de résultat entre les deux études est due principalement aux échantillons utilisés plutôt qu'aux méthodologies employées.

Plus récemment, Wu (1997) observe une manipulation à la baisse des résultats l'année qui précède la réalisation d'un MBO, en utilisant une méthodologie différente de celles de DeAngelo (1986) et de Perry et Williams (1994). Wu (1997) considère que si l'on observe une baisse systématique du résultat ${ }^{5}$ avant un MBO, elle est le fruit d'une gestion des résultats. Par ailleurs, il remarque qu'un certain nombre de $\mathrm{MBO}$ sont proposés par des dirigeants de sociétés après le lancement d'une offre publique hostile sur cette dernière. Dans ce cas, le MBO est une technique de défense anti-OPA et ne permet pas à ces derniers de disposer du temps nécessaire à une gestion à la baisse des résultats. En reprenant l'échantillon de DeAngelo, et en excluant les sociétés ayant fait l'objet d'une offre hostile, il observe une manipulation à la baisse des résultats, contredisant les conclusions de DeAngelo.

Enfin, sur le marché français, Le Nadant (1999) étudie 118 LBO dont $60 \mathrm{MBO}$. Elle ne parvient pas à mettre en évidence une manipulation des résultats.

\section{3. Étude empirique}

Il s'agit ici de tester l'hypothèse selon laquelle les sociétés initiatrices d'une offre de renforcement ou de fermeture manipulent à la baisse le résultat comptable de leur filiale afin de proposer aux actionnaires minoritaires un prix d'offre plus faible.

\subsection{Les données de l'étude empirique}

Cette étude est fondée sur les offres publiques de renforcement ou de fermeture du capital ayant eu lieu entre 1994 et 1997. Ces offres comprennent les offres publiques simplifiées (OPS) qui correspondent aux offres réalisées par un initiateur détenant déjà la moitié du capital ou des droits de vote de la société visée, ainsi que les offres publiques de retrait (OPR) ${ }^{6}$ et les retraits obligatoires (RO) qui ont eu lieu lorsque l'initiateur détient $95 \%$ du capital de la cible.

De cet échantillon initial, les offres en titres (OPE) ont été exclues. L'exclusion de ces offres se justifie par le fait que dans cette situation, la gestion des résultats peut également se faire au niveau de la société initiatrice de l'offre, risquant d'introduire un biais dans les résultats obtenus. Une manipulation du résultat de l'initiateur à la hausse permet de rendre les parités d'échange plus favorables au lanceur de l'offre et d'opérer un transfert de richesse au détriment des actionnaires de la cible. Ceci est confirmé empiriquement par Erickson et Wang (1999) qui observent que les sociétés qui rémunèrent une prise de contrôle par échange de titres pratiquent une gestion à la hausse de leur résultat trimestriel afin d'accroître leur cours boursier. Les sociétés qui ont fait l'objet d'une prise de contrôle l'année précédant l'offre ont également été exclues. Dans ce cas, l'initiateur de l'offre n'a pu disposer du temps nécessaire à une gestion du résultat et la société n'entrait donc pas dans le champ de cette étude. Ceci concerne les offres qui ont été précédées d'une OPA, d'une OPE ou d'une cession de bloc ayant entraîné une garantie de cours. Lorsqu'une même société a été successivement l'objet d'une offre simplifiée et d'une offre de retrait dans un délai d'un an, seule la première opération a été retenue. Enfin, pour qu'une société soit incluse dans l'échantillon, il est nécessaire de disposer de sa note d'information et de ses comptes consolidés sur trois exercices. Ces deux derniers critères de sélection ont 
conduit à exclure un nombre élevé de sociétés (77). Cette difficulté à obtenir de l'information s'explique par le fait qu'une proportion importante des sociétés entrant dans le champ de l'étude sont cotées sur le hors-cote et ne sont donc pas tenues à une obligation de publication au BALO. L'échantillon final est composé de 95 sociétés, se répartissant en 24 OPAS, 8 OPR et 63 RO.

Ces opérations interviennent sur les différents marchés de cotation avec une tendance nette pour les retraits obligatoires (RO) à avoir principalement lieu sur le marché libre ${ }^{7}$, ce qui s'explique par le faible flottant des titres concernés. Cette technique a permis dans un premier temps de nettoyer le hors-cote avant sa disparition le 2 juillet 1998. Elle est désormais utilisée massivement sur le premier marché, les RO faisant très souvent suite à une OPA simplifiée. Dans cette situation, les opérations s'inscrivent dans une continuité, l'éventualité d'un RO étant évoquée dans la note d'information de l'offre simplifiée (tableau 1).

\section{Tableau 1}

Marché de cotation des cibles

\begin{tabular}{|l|c:c:c|}
\cline { 2 - 4 } \multicolumn{1}{c|}{} & OPAS & OPR & RO \\
\hline Règlement mensuel & 7 & 1 & 0 \\
Comptant & 10 & 6 & 15 \\
Second marché & 6 & 1 & 6 \\
Hors cote & 1 & 0 & 42 \\
\hdashline$\cdots \cdots \cdots$ & -3 & 63 \\
\hline
\end{tabular}

Des différences très importantes existent entre les cibles, comme en atteste le tableau 2. Cela se traduit notamment par des écarts élevés entre les moyennes et les médianes, que ce soit en termes de capitalisation boursière, d'actif ou de soldes de gestion.

Tableau 2

Statistiques descriptives des sociétés cibles étudiées (KF)

\begin{tabular}{|l|c|r|r|r|}
\cline { 2 - 5 } \multicolumn{1}{c|}{} & MoYenNe & Médiane & \multicolumn{1}{c|}{ Minimum } & \multicolumn{1}{c|}{ Maximum } \\
\hline Capitalisation boursière & 1721356 & 640543 & 21820 & 14559151 \\
Actif net total & 7055813 & 2159049 & 46068 & 114541000 \\
Chiffre d'affaires & 5158641 & 1773726 & 3000 & 52572200 \\
Résultat net & 29687 & 15457 & -2912000 & 3250000 \\
Excédent brut d'exploitation & 471430 & 156569 & -524800 & 8237000 \\
\hline
\end{tabular}

Statistiques concernant l'exercice dont les états financiers servent à l'appréciation du prix d'offre.

\subsection{Mise en évidence d'une manipulation et hypothèses alternatives}

\subsection{MESURE DE LA GESTION DES RÉSULTATS}

Dans un premier temps, les recherches en matière de gestion des résultats se sont intéressées aux choix de méthodes comptables et à leur changement. L'idée en est que les dirigeants optent, lorsqu'ils en ont la possibilité, pour des méthodes comptables qui leur permettent d'atteindre le niveau de résultat souhaité. Parmi les options qui s'offrent à eux, on retrouve notamment les méthodes d'évaluation des stocks (LIFO versus FIFO), les méthodes d'amortissement (linéaire versus dégressif) ainsi que les durées d'amortissement, tout particulièrement en ce qui concerne les actifs incorporels (marques, 
goodwill). Ce type de recherche a vite montré ses limites du fait même de la faible latitude dont disposent les dirigeants dans ce domaine et de l'impossibilité de changer continuellement de méthodes. Ces pratiques sont, par ailleurs, particulièrement visibles et il est peu probable qu'elles soient mises en œuvre dans le cadre d'une offre publique de renforcement ou de fermeture du capital. Ceci a conduit à un renouvellement des méthodologies utilisées et notamment au recours à l'étude des accruals ${ }^{8}$.

Les accruals ou variables comptables de régularisation (VCR) représentent la différence entre le résultat net d'un exercice et les flux de trésorerie générés par l'activité de l'entreprise au cours de la même période. Cette différence résulte du choix d'une comptabilité d'engagement au détriment d'une comptabilité de caisse, c'est-à-dire de la prise en compte pour la détermination du résultat des produits non encaissables ou non encaissés et des charges non décaissables ou non décaissées. Il faut encore y ajouter la production immobilisée qui résulte d'un simple jeu d'écritures (Chalayer, 1995). Les VCR « correspondent donc aux produits et charges qui n'ont pas généré un flux de trésorerie " (Chalayer et Dumontier, 1996).

\section{VCR = Résultat - Flux de trésorerie d'exploitation}

À la différence des États-Unis où la publication de ce dernier solde (flux de trésorerie) est obligatoire, les entreprises françaises n'y sont pas soumises. Dans le contexte français, il est possible d'estimer le montant des VCR par le biais de leurs éléments constitutifs, c'est-à-dire la variation du besoin en fonds de roulement et la production immobilisée, déduction faite des dotations aux amortissements et provisions nettes des reprises.

\section{$\mathrm{VCR}=\mathrm{RAP}-\mathrm{DAP}+\triangle \mathrm{BFR}+$ Production immobilisée}

Comme l'a montré DeAngelo (1986), les VCR sont composées d'une partie non discrétionnaire sur laquelle les dirigeants ne peuvent influer et d'une part discrétionnaire qui résulte des décisions de ces derniers afin d'atteindre le niveau de résultat souhaité.

avec: $\mathrm{VCR}_{\mathrm{T}}$ : VCR totales,

$$
\mathrm{VCR}_{\mathrm{T}}=\mathrm{VCR}_{\mathrm{D}}+\mathrm{VCR}_{\mathrm{ND}}
$$

$V_{C C R}$ : VCR discrétionnaires,

$\mathrm{VCR}_{\mathrm{ND}}$ : VCR non discrétionnaires.

La part non discrétionnaire résulte d'une politique normale d'amortissement des immobilisations et de provisionnement des dépréciations, risques et charges. Il en va de même du niveau du BFR qui est directement lié à l'activité de l'entreprise et à son évolution. La part discrétionnaire des VCR résulte, quant à elle, des choix des dirigeants afin de majorer ou de minorer le résultat d'un exercice. Elle ne peut être observée directement et fait l'objet d'une estimation par différence entre le montant des VCR totales et celui des VCR non discrétionnaires.

Le problème qui se pose est celui de l'estimation de la part non discrétionnaire des VCR. Pour ce faire, la plupart des études utilisent des modèles régressifs sur séries temporelles ou en coupe instantanée. Dans le cadre de l'étude, cette méthodologie n'a pas été retenue. Ce choix est motivé par deux raisons.

Tout d'abord, la validité statistique des modèles régressifs, tels que ceux de Jones (1991) ou de ses nombreux dérivés sur séries temporelles, est très variable. Le coefficient de détermination moyen obtenu par Jones (1991) est de $23 \%$, de 50,9 \% pour Perry et Williams (1994) ${ }^{9}$ et de 52,7 \% pour Easterwood (1998) qui utilisent des données trimestrielles, augmentant par là même le nombre d'observations par société. Sur le marché français, l'utilisation de modèles régressifs sur données chronologiques semble hasardeuse dans le cas des comptes consolidés, aucune base de données ne remontant avant 1986, date de l'obligation pour les sociétés cotées d'établir des comptes consolidés ${ }^{10}$. Or, il 
paraît difficile de réaliser une régression multiple sur moins d'une dizaine d'observations et d'obtenir des résultats statistiquement significatifs.

Plus récemment, des études telles que celles de Kasznik (1999) et Jeter et Shivakumar (1997) ont montré que les modèles régressifs conduisaient à de meilleurs résultats lorsqu'ils étaient appliqués non plus à des données temporelles mais sur des données en coupe instantanée de portefeuilles sectoriels. Cette méthodologie paraît, elle aussi, difficilement applicable au cas français du fait de l'absence de normalisation dans la présentation des comptes consolidés. La mise en œuvre de ces modèles requiert l'utilisation d'informations comptables relativement fines, telles que les reprises sur amortissements et provisions, données qui sont fréquemment absentes dans les rapports annuels des sociétés cotées, tout particulièrement lorsque ces dernières sont cotées sur le second marché ou le hors-cote " mentalement, elle nécessite la constitution de portefeuilles sectoriels d'au moins une dizaine d'observations pour être statistiquement significative. Dans le cas présent, du fait du nombre limité de sociétés cotées en France ainsi que de l'importance de celles répertoriées dans l'étude sous le code APE 760 (holdings), ceci s'est révélé impossible.

La possibilité de travailler sur des secteurs élargis a été envisagée, notamment sur la base de la décomposition du SBF 250 en douze activités. Le problème de l'homogénéité des entreprises au sein d'un même secteur s'est alors posé. Pour que le $\mathrm{R}^{2}$ de la régression en coupe instantanée soit suffisamment élevé, il est impératif que les sociétés au sein d'un même secteur aient une structure d'actifs proche. Au sein du secteur 106 (autres biens de consommation), il est peu probable qu'il en soit ainsi entre Essilor et Hermès ou entre Carrefour et Naf-Naf (secteur 208 : Distribution). Par ailleurs, au sein du code 312 (sociétés d'investissements), la structure de l'actif des comptes consolidés n'est pas liée à l'activité mais au portefeuille de participations, ce qui rend impossible la mise en œuvre d'une régression à l'intérieur de ce secteur. Cette critique de la méthode de Jones en coupe instantanée s'applique également, selon Bernard et Skinner (1996), aux études empiriques américaines.

L'impossibilité d'obtenir des données consolidées fiables et détaillées ainsi que la validité statistique très variable des modèles régressifs, qu'ils soient sur séries temporelles ou en coupe instantanée, ont conduit à s'inspirer de la méthodologie développée par Wu (1997) dans le cadre des MBO. Selon Wu (1997), en se plaçant dans une optique "darwinienne ", les entreprises ne devraient pas connaître de baisse systématique de leurs résultats. Il en déduit qu'une diminution systématique du résultat des sociétés cibles lors de l'exercice qui précède un MBO est supposée être la conséquence d'une gestion à la baisse des résultats. Létude du résultat net présente par ailleurs l'avantage de synthétiser les conséquences des différentes techniques de gestion des bénéfices, qu'il s'agisse des changements de méthodes comptables, de la gestion des VCR ou de la gestion dans le temps des ventes, et est donc mieux à même de détecter une éventuelle manipulation.

\section{CHOIX DE LA PÉRIODE D'EVEENEMENT}

L'hypothèse qui est faite dans le cadre de cette recherche est que le résultat de la société cible est manipulé lors de l'exercice dont les comptes annuels servent à l'appréciation du prix d'offre. Ce choix est différent de la plupart des études nord-américaines qui considèrent que la manipulation a systématiquement lieu l'année précédant l'opération. Or, dans un nombre important de cas, à la date de lancement de l'offre, les comptes annuels de l'exercice précédent ne sont pas connus, ce qui impose de recourir aux comptes annuels de l'avant-dernier exercice pour évaluer la cible. Le choix de la période d'événement a donc été effectué sur la base de l'exercice de référence utilisé dans la note d'information. 


\section{Résultats de l'étude empirique}

\section{1. Étude du résultat net et des variations du résultat net}

Le résultat net de la société cible est standardisé par le montant de la capitalisation boursière en début d'exercice. Bien que les cours boursiers soient peu pertinents en matière de valorisation lorsque le flottant est faible, il a semblé qu'il s'agissait là du meilleur déflateur, notamment par rapport aux capitaux propres en valeur comptable. En effet, cette valeur comptable des capitaux propres ne prend pas en compte les possibles options de croissance de la société et est, dans certains cas, négative ${ }^{12}$. Un test non paramétrique de Wilcoxon sur les rangs est utilisé afin d'observer si les résultats nets comptables sur les trois exercices qui précèdent l'offre sont significativement différents d'une année à l'autre. Ce test est préféré à celui de Student qui requiert une distribution normale des données, qui n'est pas vérifiée dans le cas présent. Puis la méthodologie de Wu (1997) est répliquée. Wu (1997) s'intéresse non pas aux différences de résultats entre deux périodes mais aux différences de variation de résultats entre deux exercices. Cette différence entre $\mathrm{N}$ et $\mathrm{N}-1$ est standardisée par la capitalisation boursière à la fin de l'exercice N-1. Dans ce cas, l'hypothèse testée est celle d'une différence nulle de variation du résultat net standardisé. L'idée est que les sociétés doivent connaître, en moyenne, un taux de croissance constant de leurs résultats. Si les variations d'une année sont systématiquement moindres que celles de l'année précédente, il est possible de faire l'hypothèse qu'il y a eu manipulation.

Le tableau 3 présente les résultats de cette étude.

\section{Tableau 3}

Résultat net, variation du résultat net et capitalisation boursière

\begin{tabular}{|c|c|c|}
\hline & $N-1$ & $\mathbf{N}$ \\
\hline \multicolumn{3}{|l|}{ Résultat net standardisé } \\
\hline Nombre d'observations & 95 & 95 \\
\hline Médiane (\%) & 6,90 & 4,06 \\
\hline Moyenne (\%) & 4,14 & $-18,67$ \\
\hline Négatifs : positifs & $19: 66$ & $32: 63$ \\
\hline Test de Wilcoxon (seuil) a & 0,629 & $* * * 0,001$ \\
\hline \multicolumn{3}{|l|}{$\Delta$ Résultat net standardisé } \\
\hline Nombre d'observations & 83 & 95 \\
\hline Médiane (\%) & 0,27 & $-0,83$ \\
\hline Moyenne (\%) & 3,11 & $-15,25$ \\
\hline Négatifs : positifs & $40: 42$ & $56: 39$ \\
\hline Test de Wilcoxon (seuil) & 0,686 & *0,070 \\
\hline \multicolumn{3}{|l|}{ Capitalisation boursière " } \\
\hline Nombre d'observations & 95 & 95 \\
\hline Médiane & 563715 & 640543 \\
\hline Moyenne & 1595963 & 1721356 \\
\hline Test de Wilcoxon (seuil) & 0,473 & $* * * 0,010$ \\
\hline
\end{tabular}


Les tests effectués mettent en évidence une gestion à la baisse des résultats des sociétés cibles d'une offre publique l'année dont les états financiers servent de base à l'appréciation du prix d'offre dans la note d'information (année $\mathrm{N}$ ). Cetre gestion s'apprécie au travers des différences de résultats nets et de variations de résultats nets.

Le résultat net standardisé moyen (médian) passe de 4,14\% (6,90\%) de la capitalisation boursière $\grave{a}-18,67 \%(4,06 \%)$ entre $N-1$ et $N$. Cette baisse est statistiquement significative au seuil de $0,1 \%$, allant dans le sens d'une manipulation du résultat comptable de la cible. L'année qui précède, la différence n'est pas significative, le résultat net moyen (médian) étant de 4,4\% (7,06\%) en N-2 et de $4,14 \%(6,90 \%)$ en N-1. Ceci peut se justifier par le fait que les dirigeants n'ont aucun intérêt à gérer à la baisse les résultats de façon précoce car une gestion à la baisse une année risque d'induire un effet mécanique à la hausse sur le résultat de l'exercice suivant. Cette différence de résultat net entre $\mathrm{N}$ et $\mathrm{N}-1$ pourrait s'expliquer par un accroissement de la capitalisation boursière. Cependant, elle reste significative lorsque la base de standardisation est commune aux deux années ${ }^{13}$.

L'étude des variations de résultat va également dans le sens de l'hypothèse d'une gestion à la baisse $\mathrm{du}$ résultat net. Le taux de croissance du résultat est négatif, en moyenne (médiane) de $-15,25 \%$ $(-0,8 \%)$ contre une croissance positive l'année précédente de $3,1 \%(0,27 \%)$. En $\mathrm{N}-1$, la variation du résultat standardisé n'est pas significativement différente de celle de $\mathrm{N}-2$.

Les enjeux financiers étant très différents entre les OPAS et les OPR et RO, il est possible que les cibles d'une offre simplifiée soient plus susceptibles de faire l'objet d'une gestion de leur résultat. La décomposition des résultats par type d'offre conduit à des résultats contrastés selon que l'on s'intéresse au résultat net ou à la variation du résultat net. Dans le premier cas, seuls les RO font apparaître une gestion des résultats, significative au seuil de $0,7 \%$. Le résultat baisse pour 44 sociétés sur un total de 63. En revanche, lorsque l'on s'intéresse aux variations du résultat, la décomposition ne fait pas ressortir de différence significative contrairement au résultat observé sur l'échantillon dans sa totalité. Il faut cependant noter que l'étroitesse des sous-échantillons limite la pertinence des conclusions qu'il est possible de tirer de leur étude.

\section{2. Étude des variables comptables de régularisation}

Afin d'affiner les résultats obtenus, les deux principaux éléments constitutifs des VCR ont été étudiés, à savoir les dotations aux amortissements et provisions nettes des reprises (DAP) ainsi que la variation du BFR. En préalable, il convient de noter que ces informations proviennent de comptes consolidés ${ }^{14}$ et ne font donc pas l'objet d'une présentation normalisée, à la différence des comptes sociaux. De ceci, il ressort principalement qu'il n'a pas été possible de distinguer les dotations aux amortissements des dotations aux provisions. Ceci constitue une perte d'information non négligeable, de même qu'il aurait été particulièrement instructif de pouvoir distinguer selon que les provisions étaient d'exploitation, financières ou exceptionnelles. Dans le cadre de cette étude, la méthodologie de DeAngelo (1986) est reprise en y apportant une amélioration. DeAngelo (1986) considère que toutes les variations des VCR sont discrétionnaires, sans tenir compte de l'effet que peuvent avoir les modifications de l'environnement de l'entreprise sur leur niveau. Afin de tenir compte de l'impact des conditions économiques sur les VCR, elles ont été standardisées par les éléments au sein desquels elles trouvent leur origine. Ainsi, les DAP nettes des reprises sont standardisées par le montant total de l'actif brut hors disponibilités et la variation du BFR par le montant du chiffre d'affaires de l'année. Il faut cepen- 
dant noter, comme l'ont montré Dechow, Sloan et Sweeney (1995), que les dirigeants ont également la possibilité de gérer dans le temps les ventes afin d'influer sur le résultat. Si tel est le cas, la diminution du chiffre d'affaires entraîne, toutes choses égales par ailleurs, une réduction du résultat net. Pour ce qui est du total des VCR, il est standardisé par le montant de l'actif net total.

\section{Tableau 4}

Étude des VCR

\begin{tabular}{|c|c|c|}
\hline & $N-1$ & $N^{b}$ \\
\hline \multicolumn{3}{|l|}{ Chiffre d'affaires (KF) } \\
\hline - moyen & 5210016 & 5158641 \\
\hline - médian & 2008537 & 1773726 \\
\hline - test de Wilcoxon (seuil)a & & $* 0,068$ \\
\hline \multicolumn{3}{|l|}{ VCR standardisées } \\
\hline - moyen & $-0,057$ & $-0,079$ \\
\hline - médian & $-0,041$ & $-0,052$ \\
\hline - test de Wilcoxon (seuil)a & & 0,208 \\
\hline \multicolumn{3}{|l|}{ DAP standardisées } \\
\hline - moyen & 0,0340 & 0,0470 \\
\hline - médian & 0,0344 & 0,0343 \\
\hline \multicolumn{3}{|l|}{$\triangle$ BFR standardisée } \\
\hline - moyen & $-0,0354$ & $-0,0656$ \\
\hline - médian & $-0,0004$ & $-0,0068$ \\
\hline - test de Wilcoxon (seuil)a & & 0,505 \\
\hline \multicolumn{3}{|l|}{ EBE/CA } \\
\hline - moyen & 0,0716 & 0,0923 \\
\hline - médian & 0,0640 & 0,0529 \\
\hline - test de Wilc & & 0,900 \\
\hline
\end{tabular}

a : différence significative par rapport à l'année précédente au seuil de $1 \%\left(^{* * *}\right), 5 \%\left(^{* *}\right)$ ou $10 \%\left({ }^{*}\right)$.

$b: N$ correspond à l'année dont les états financiers figurent dans la note d'information (année de la possible manipulation).

L'étude approfondie des données comptables montre que les sociétés cibles connaissent un accroissement de leur chiffre d'affaires, significatif au seuil de $6,8 \%$ pour le test sur les rangs de Wilcoxon. Ceci va dans le sens de l'hypothèse d'une manipulation comptable puisque, dans le même temps, le résultat net baisse ${ }^{15}$. Les VCR standardisées diminuent et passent, en moyenne, de $-0,057 \grave{a}-0,079$, allant dans le sens de l'hypothèse testée. Cependant, cette baisse n'est pas statistiquement significative aux seuils conventionnels. Concernant les DAP nettes des reprises et la variation du BFR, aucune différence statistiquement significative aux seuils conventionnels n'est observée en utilisant les mêmes tests.

La stabilité du taux de marge brute d'exploitation (EBE/CA), alors que le résultat net diminue, qu'il soit standardisé par la capitalisation boursière ou le chiffre d'affaires, renforce l'hypothèse d'une 
gestion à la baisse des résultats. La décomposition des résultats des tests statistiques par type d'offres n'apporte aucun résultat supplémentaire.

La baisse significative du résultat net des cibles d'une offre publique de renforcement du contrôle ou de fermeture du capital, accompagnée d'une augmentation de leur chiffre d'affaires et d'un maintien de leur taux de marge brute, semble traduire une manipulation de leur résultat. Cette conclusion est renforcée par le fait que les sociétés observées n'évoluent pas dans des secteurs en très forte croissance, pour lesquels il est courant d'observer en parallèle une augmentation du chiffre d'affaires et une dégradation du résultat.

\subsection{Hypothèses alternatives}

Cette mise en évidence d'une gestion à la baisse des résultats, sans qu'il soit cependant possible de l'expliquer par la manipulation des VCR, conduit à s'interroger sur les autres possibilités qui s'offrent à l'initiateur pour réduire le résultat comptable afin de proposer un prix d'offre plus faible. Trois hypothèses alternatives ont été identifiées.

\subsubsection{GESTION DE LA DATE DE DÉROULEMENT DE L'OPÉRATION}

La première possibilité consiste pour l'initiateur à choisir le moment le plus opportun pour lancer une offre publique sur l'une de ses filiales, par exemple après l'annonce de résultats médiocres ou lorsque cette dernière se trouve dans une situation économique et financière délicate. Dans ce cas, la société mère est mieux informée que le marché sur le potentiel de redressement de sa filiale. Elle est donc mieux à même de valoriser correctement l'entreprise et peut gérer de façon opportuniste la date d'annonce du lancement de l'offre. Ce comportement opportuniste pourrait expliquer le fait que les résultats des sociétés cibles soient plus faibles en $\mathrm{N}$ qu'en $\mathrm{N}-1$. Cette hypothèse est cependant en contradiction avec l'augmentation significative du CA l'année précédant l'offre, sauf à supposer une réduction systématique des marges des cibles.

\subsection{GESTION INTRA-GROUPE DES RÉSULTATS}

Un second type de gestion " réelle " peut résulter des liens qui unissent la société initiatrice et la cible. $\mathrm{Du}$ fait de son niveau de participation, les relations entre les deux sociétés ne sont pas uniquement capitalistiques mais peuvent également être commerciales. Dès lors, il convient de s'interroger sur le lien de dépendance économique de la cible vis-à-vis de l'initiateur et sur les possibilités de ce dernier d'influer sur les résultats de sa filiale dans le cadre de cette relation commerciale, et notamment au travers des prix de transfert. Ces prix de transfert permettent de localiser le bénéfice dans certaines sociétés au détriment d'autres par le biais des opérations intra-groupe. Dans le cas des offres publiques, si la filiale entretient des relations commerciales au sein du groupe, son résultat peut être géré à la baisse en lui faisant acheter des produits à un prix plus élevé ou vendre sa production à un prix plus bas. Au niveau empirique, ce type de pratique semble difficile à mettre en évidence.

\section{EFFET TAILLE DES DOTATIONS AUX AMORTISSEMENTS ET PROVISIONS}

Les comptes consolidés des sociétés cotées françaises ne distinguent pas, dans leur très grande majorité, les dotations aux provisions des dotations aux amortissements. Or il s'agit là du principal poste 
manipulable dans le cadre d'une gestion des résultats, contrairement aux dotations aux amortissements dont le montant résulte d'un plan déterminé à l'avance et fortement contraint par les dispositions fiscales. Si les dotations aux amortissements représentent une part importante des dotations totales, les tests statistiques peuvent se révéler incapables de détecter la manipulation des provisions qui se retrouvent " noyées ". De même, l'importance des dotations totales (amortissements et provisions) par rapport au résultat de l'exercice peut faire qu'une variation très faible de leur montant aura un impact de premier ordre sur le résultat net de l'exercice. Cet effet taille pourrait expliquer l'absence de différence significative observée tant au niveau des VCR totales que des DAP.

Voir l'annexe 1 pour une présentation détaillée de cette hypothèse.

\section{Conclusion}

Cette étude a permis de mettre en évidence une gestion à la baisse des résultats des sociétés qui sont la cible d'une offre publique de renforcement ou de fermeture du capital. Une augmentation statistiquement significative du chiffre d'affaires, accompagnée d'un taux de marge brute d'exploitation constant et d'une baisse du résultat net, nous conduit à cette conclusion. Sur les moyens de cette manipulation, les résultats obtenus ne permettent pas de conclure de façon nette en étudiant les VCR, du fait notamment de problèmes liés à la qualité de l'information disponible et à sa précision. Ceci a conduit à émettre des hypothèses alternatives qui pourraient expliquer cette baisse du résultat qui ne trouve pas sa traduction dans l'étude des VCR. Il s'agit notamment de l'hypothèse d'une gestion des résultats non plus comptable mais « réelle », par le biais des relations commerciales qui peuvent exister entre les différentes sociétés du groupe. Ceci n'exclut pas pour autant le recours à une gestion des VCR dans certains cas. Enfin, il faut noter que les agrégats comptables utilisés peuvent expliquer l'absence de résultat statistiquement significatif, l'effet taille des dotations aux amortissements et provisions biaisant, à notre avis, les résultats obtenus. Il ne faut pas non plus exclure l'hypothèse d'un comportement opportuniste en matière de choix de la date de déroulement de l'opération, les initiateurs pouvant attendre le moment le plus opportun pour lancer l'offre.

L'objet de cette manipulation est double. Tout d'abord, elle influe sur le résultat de la mise en œuvre des méthodes d'évaluation dites analogiques, qui sont fondées sur l'utilisation de multiples du résultat comptable. Mais son objet est également de faire baisser le cours boursier en faisant parvenir au marché un signal biaisé quant à la valeur de la société.

Cette gestion conduit à un transfert de richesse qui s'opère au détriment des minoritaires. Elle illustre le conflit d'intérêt qui existe entre les dirigeants des sociétés cotées et les actionnaires minoritaires, conflit qui a conduit ces dernières années à un renforcement de la législation (présence obligatoire dans certains cas d'un expert indépendant) et à un développement des associations de petits porteurs.

Cette étude a été l'occasion de mettre en œuvre une méthodologie plus simple que les modèles régressifs. Le constat d'une gestion baissière des résultats ne peut que renforcer la nécessité d'un examen plus strict du prix d'offre, que ce soit de la part des autorités du marché - COB et CMF ou des experts qui sont amenés à se prononcer sur l'équité du prix proposé. Ceci doit également conduire ces derniers à repenser leur mission qui ne doit pas se limiter à vérifier la pertinence des méthodes d'évaluation employées mais qui doit aller au-delà, quitte à se substituer, pour partie, à la mission jusque-là dévolue aux commissaires aux comptes. 


\section{Annexe 1}

Afin de tester l'hypothèse d'un effet taille des DAP, nous avons agrégé les comptes sociaux (en KF) des sociétés dont le chiffre d'affaires était supérieur à 2 milliards de francs à partir de la base de données DIANE (583 sociétés pour 1997). Le choix des comptes sociaux au détriment des comptes consolidés s'explique par notre objectif qui est de faire ressortir le plus finement possible la structure des comptes de résultat. En effet, les comptes sociaux tels qu'ils figurent dans DIANE sont issus de la liasse fiscale, ce qui leur confère une qualité et une précision que n'ont pas les comptes consolidés.

\section{DÉCOMPOSITION DES CHARGES ET PRODUITS CALCULÉS EN KF (CUMUL DE 583 SOCIETTES)}

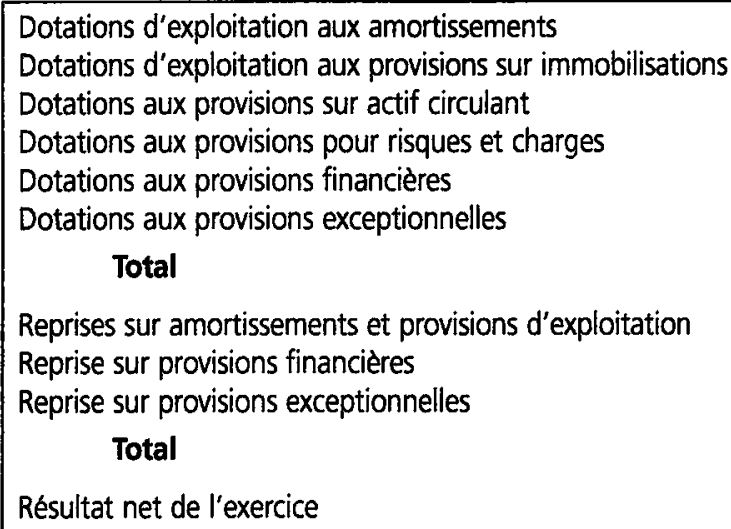

194624096

13312583

18611066

84628360

46877832

92385088

450439025

125905776

18325592

71157456

215388824

85840112

Le montant total cumulé des dotations pour l'année 1997 s'élève à 450 milliards de francs pour un résultat net cumulé de 85 milliards, soit un rapport de 5,2. Une augmentation de $1 \%$ des dotations entraine une chute de $5,2 \%$ du résultat de l'exercice. Cette différence pourrait expliquer l'absence de résultat statistiquement significatif lorsque le test de Wilcoxon est utilisé. Quant au solde net (DAP - RAP), il s'élève à 235 milliards, soit 2,73 fois le résultat cumulé. L'effet multiplicateur sur le résultat net des manipulations des VCR calculées leur confère ainsi un grand intérêt dans le cadre d'une gestion des résultats.

\section{Notes}

1. Pour une revue de la littérature, Watts et Zimmerman (1986, 1990), Raffournier (1990) et Dumontier et Raffournier (1999).

2. Code de Commerce, art. 10.

3. Sur l'importance de la communication financière pour l'évaluation par le marché, voir Saada (1994).

4. Management Buy Out.

5. Et non plus des accruals comme chez DeAngelo (1986) ou Perry et Williams (1994).

6. Nous avons exclu de notre étude les OPR qui résultaient de la transformation d'une société en commandite par actions lorsque les majoritaires détiennent plus des deux tiers des droits de vote (art. 5.5.4.) ou d'une modification significative des statuts ou de l'activité (art. 5.5.5.).
7. Marché hors-cote jusqu'à sa disparition en juillet 1998.

8. Variables comptables de régularisation, selon la terminologie de Le Nadant (1999).

9. Mais moins de la moitié des coefficients des régressions multiples sont significatifs aux seuils conventionnels.

10. Loi du 3 juillet 1985 .

11. Cette information est souvent agrégée dans les lignes « autres produits d'exploitation ", «produits financiers" ou " produits exceptionnels ".

12. En standardisant par le chiffre d'affaires, les résultats pour le test de Wilcoxon sont identiques.

13. La base retenue est la capitalisation au début de l'année $\mathrm{N}$.

14. Les données ont été collectées sur les notes d'information ainsi que sur la base de données DIANE. 
15. La baisse du chiffre d'affaires moyen est due à quelques observations extrêmes, la majorité des sociétés connaissant une augmentation de leur CA.

\section{Bibliographie}

BALl R., BROWN P. (1968), «An Empirical Evaluation of Accounting Income Numbers ", Journal of Accounting Research, vol. 6, automne, p. 159-178.

BEAVER W. (1968), "The Information Content of Annual Earnings Announcements ", Journal of Accounting Research, vol. 6, p. 67-92.

BERNARd V., SKINNER D. (1996), "What Motivates Managers' Choice of Discretionary Accruals ?", Journal of Accounting and Economics, vol. 22, p. 313-325.

CHALAYER S. (1995), " La manipulation des variables comptables à des fins de lissage des résultats ", Actes du XVI congrès de l'Association française de comptabilité, Montpellier, p. 1208-1222.

Chalayer S., Dumontier P. (1996), " Performances économiques et manipulations comptables ; une approche empirique ", Actes du XVII congrès de l'Association française de comptabilité, p. 803-818.

Chen C., Lin J.W., Sauer D. (1997), "Earnings Announcements, Quality and Quantity of Information, and Stock Price Changes ", Journal of Financial Research, vol. 20, p. 483-503.

Christie A., Zimmerman J. (1994), " Efficient and Opportunistic Choices of Accounting Procedures : Corporate Control Contests ", The Accounting Review, vol. 69, p. 539-566.

CORMIER D., MAGNAN M. (1995), "La gestion stratégique des résultats : le cas des firmes publiant des prévisions lors d'un premier appel public à l'épargne ", Comptabilité-Contrôle-Audit, vol. 1, p. 45-61.

DeAngelo H., DeAngelo L., Rice E. (1984), " Going Private : Minority Freezeouts and Stockholder Wealth ", Journal of Law and Economics, vol. 27, p. 367-401.

DeANGelo L. (1986), "Accounting Number as Market Valuation Substitutes ", The Accounting Review, vol. 61, p. 400-420.
DeAngelo L. (1988), « Managerial Competition, Information Costs and Corporate Governance ; the Use of Accounting Performance Measures in Proxy Contests ", Journal of Accounting and Economics, vol. 10, p. 3-36.

Dechow P., SLOAN R., SweEney A. (1995), " Detecting Earnings Management ", The Accounting Review, vol. 70, p. 193-225.

Degeorge F., Patel J., Zeckhauser R. (1999), "Earnings Management to Exceed Thresholds ", Journal of Business, vol. 72, p. 1-35.

DUMONTIER P., RAFFOURNIER B. (1999), "Vingt ans de recherche positive en comptabilité financière ", Comptabilité-Contrôle-Audit mai, p. 179-197.

EASTERWOOD C. (1998), " Takeovers and Incentives for Earnings Management: an Empirical Analysis ", Joumal of Applied Business Research, vol. 14, p. 29-47.

EDDEY P., TAYLOR S. (1999), " Directors' Recommendations on Takeover Bids and the Management of Earnings : Evidence From Australian Takeovers ", Abacus, vol. 35, p. 29-45.

ERICKSON M., WANG S-W. (1999), "Earnings Management by Acquiring Firms in Stock for Stock Mergers ", Journal of Accounting and Economics, vol. 27, p. 149-176.

FAMA E. (1980), "Agency Problem and the Theory of the Firm ", Journal of Political Economy, vol. 88, p. 288-307.

Groff J., Wrught C. (1989), "The Market for Corporate Control and its Implications for Accounting Policy Choice ", Advances in Accounting, p. 3-21.

HAMON J. (1997), " Efficience faible, efficience semiforte ", Encyclopédie des marchés financiers, Simon Y. (éd.), Economica.

JeTER D., Shivakumar L. (1997), “Cross-Sectional Estimation of Abnormal Accruals Using Quartely and Annual Data : Effectiveness in Detecting Earnings Management ", Working paper \#2411997, London Business School.

JONES J. (1991), "Earnings Management During Import Relief Investigations ", Journal of Accounting Research, vol. 29, p. 193-228.

KASZNIK R. (1999), "On the Association Between Voluntary Disclosure and Earnings Management ", Journal of Accounting Research, vol. 37, p. 57-81. 
LE NADANT A.L. (1999), " La gestion des résultats comptables précédant les opérations de LBO françaises ", Comptabilité-Contrôle-Audit, tome 5, vol. 2, p. 61-82.

PATELL J.M., WOLFSON M.A. (1984), “The Intraday Speed of Adjustment of Stock Prices to Earnings and Dividend Announcements ", Journal of Financial Economics, vol. 13, p. 223-252.

Perry S., Williams T. (1994), “ Earnings Management Preceding Management Buyout Offers ", Journal of Accounting and Economics, vol. 18, p. 157-179.

RAFFOURNIER B. (1990), "La théorie positive de la comptabilité : une revue de la littérature ", Économie et Sociétés, série "Sciences de gestion ", $\mathrm{n}^{\circ} 16$, p. 137-166.

RANGAN S. (1998), "Earnings Management and the Performance of Seasoned Equity Offerings ", Journal of Financial Economics, Vol. 50, p. 101122.

SAADA T. (1994), "La communication financière de l'entreprise et son impact sur l'évaluation par le marché : une synthèse de la littérature ", Économie et Sociétés, série « Sciences de gestion ”, p. 85-112.
Subramanyam K.R. (1996), "The Pricing of Discretionary Accruals ", Journal of Accounting and Economics, vol. 22, p. 249-281.

TeOH S., WeLCH I., Wong T.J. (1998a), “ Earnings Management and the Long-Run Market Performance of Initial Public Offerings ", Journal of Finance, vol. 53, p. 1935-1974.

TeOH S., WelCh I., WONG T.J. (1998b), “ Earnings Management and the Underperformance of Seasoned Equity Offerings ", Joumal of Financial Economics, vol. 50, p. 63-99.

Thauvron A. (1998), "Étude des modes d'évaluation lors des offres publiques ", in BRECHET J.-P. et MEVELLEC P., Marchés financiers et gouvernement de l'entreprise, Presses académiques de l'Ouest.

Watts R., Zimmerman J. (1986), Positive Accounting Theory, Prentice Hall.

Watts R., Zimmerman J. (1990), " Positive Accounting Theory : a Ten Year Perspective ", The Accounting Review, vol. 65, p. 131-156.

WU Y. (1997), «Management Buyouts and Earnings Management ", Journal of Accounting, Auditing and Finance, vol. 12, p. 373-389. 